\title{
Induced Mutations in some Safflower Genotypes
}

\section{Okaz, A.M.A ${ }^{1}$; M.S. Ahmad ${ }^{2}$ and H.G.H. Sakr ${ }^{2}$}

${ }^{1}$ Agronomy Dep., Fac. of Agric., Al-Azhar Univ., Cairo, Egypt.

${ }^{2}$ Agronomy Dep., Fac. of Agric., Al-Azhar Univ., Assiut, Egypt.

Received on: 28/11/2016

Accepted for publication on: 26/12/2016

\section{Abstract}

This investigation was carried out for induce mutations in safflower (Carthamustinctorius L) at the Experimental and Research Farm, Faculty of Agriculture, AlAzhar University. The results showed access anumbers of promising mutants in M3 generation. The results shows that the Di methyl Sulfoxide (chemical mutagen) was more effective than other two treatments ( $\gamma$-ray \& electric shock), as well as the line $32\left(\mathrm{~L}_{1}\right.$ was more responsible than the other two genotypes for induction of stable promising mutants according to final results at M3especially high seed yield. The promising mutants were softness and earliness than the parental genotypes.

The earliestgeno types of flowering (116.83day) in $\mathrm{M}_{3}$ was obtained from plants of L1h1. The highest seed yield/plant (128.00 and $127.17 \mathrm{~g}$ ) was obtained from plants of $\mathrm{L}_{2} \mathrm{t}_{3}$ and $\mathrm{L}_{1} \mathrm{~h}_{2}$, respectively.

The results supported that the mutagen treatment scan be used to get new safflower genotypes is characterized by spineless, earliness and high seed yield andthus can involve in breeding program to get new varieties suitable for cultivation in reclaimed lands.

\section{Introduction:}

Safflower seeds have been found 4,000 year-old in Egyptian tombs and using by Chinese approximately 2,200 years ago. Safflower (Carthamus tinctoriusL.) is one of the important oil seed crops and has been traditionally grown for its flowers as a source of dye for coloring food and fibers. Subsequently, it is grown for edible oil, animal meal, bird feed, medicinal uses, as a potential candidate crop for production of plant made pharmaceuticals, biofuel and specialty type oils. Oil of Safflower is the richest source of linoleic acid, with average linoleic acid content around $78 \%$ of the total seed oil fatty acids (Velasco et al., 2005).

India cultivated about 0.42 million ha, produced of 0.23 million tons of seed and average productivity of $547 \mathrm{~kg} / \mathrm{ha}$, so it is consider the lead- ing producer of safflower in the world (FAOSTAT, 2006). Despite its vast potential and growth adaptability to a wide range of agro-ecological conditions, safflower remained as a neglected crop due to low seed oil content (28-36\%), spines, fiber rich seed meal and vulnerability to a number of diseases and pests. Safflower species are known to possess several desirable genes such as, drought hardiness, shattering tolerance, non-dormancy of seeds, and resistance to safflower fly, rust, and powdery mildew (Sujatha, 2007).

Artificial induction of mutations by using of physical and chemical mutagens such as radiation, chemicals and electric shock are considered to be one of the useful tools for plant improvement by increasing of genetic variability in many plant species, especially the self-fertilized plants. 
(Kharkwal, 2000; Hassan et al., 2001; Mihov et al., 2001 Wani and Anis,2001, Soliman et al., 2003), Fahmy et al., 1997; Geetha and Vaidyanathan, 1998; Hajduch et al., 1999 and Solanki and Sharma, 1999).

In Egypt, safflower area decreased year after year at Upper Egypt, because the genotypes suffering from many problems as lateness (185 days at maturity), full thorns on leaf and heads, low seed yield and low seed oil content. Therefore, the present study aimed to induce mutations for earliness, spineless and high seed yield with high oil content as a promising mutant that could be used in breeding program to get new varieties.

\section{Materials and Methods:}

Three mutagens i.e. gamma ray $(\gamma$-ray) and Di methyl Sulfoxide and electric shock were used for induction of mutation on three safflower genotypes (Line32, Line37 and Line40) during three seasons 2013/2014, 2014/2015 and 2015/2016. The gamma ray doses were $10 \mathrm{kr}$ and $20 \mathrm{kr}$ while the concentrations of Di methyl Sulfoxide were (1000 ppm), (2000ppm) and (3000ppm). The electric shock in the presence of the used chemical solutions as follows: Monosodium phosphate (30000 ppm/liter), Monosodium phosphate $(50000 \mathrm{ppm} / \mathrm{liter})$ and Sodium nitrate (50000 ppm/liter).

Three safflower lines; Line 32, line 37 and Line 40 were obtained from Oil Crop Research Section, Field Crop Research Institute, Agricultural Re- search Center (ARC) were used in this study. The selected variants at the present study included apparent morphological characters, especially earliness and softness change, as well as the change in seed yield attribute characters. These variants were screened to isolate M1 and M2 generations. These mutants characterized with, thorns leaves, flowering date (earliness, lateness), and high seed weight. In M3 generation the stable M2 mutant lines were screened and recorded, especially these possessed softness and earliness.

\section{Gamma ray:}

40 grams from seeds from each line were backed in paper bags and subjected to gamma ray doses of 10 $\mathrm{Kr}(\mathrm{r} 1)$ and $20 \mathrm{Kr}(\mathrm{r} 2)$ and the exposure time was 30 minutes in October, 2013 in Middle Eastern Regional Radioisotope Center for the Arab countries at NRC, Dokki, Cairo, Egypt.

\section{Di methyl Sulfoxide:}

40 grams from the seeds from each line were soaked in prepared aqueous solution of Di methyl Sulfoxide (DMS) of three different concentrations (1000 ppm (h1), 2000 ppm (h2) and $3000 \mathrm{ppm}(\mathrm{h} 3)$ for 24 hours.

\section{Electric shock:}

40 grams from seeds from each line were germinated and exposed to electric shock inside special electric analysis set to invent the DNA activity through the cell division during germination of the seeds for mutations induction (Ahmad 2011). 
The used chemical solutions were as follow:

\begin{tabular}{|c|l|c|c|}
\hline No & \multicolumn{1}{|c|}{ Chemical components } & Concentration & Brief \\
\hline 1 & Monosodium phosphate $\mathrm{NaH}_{2}\left(\mathrm{PO}_{4}\right)_{3}$ & $(50000 \mathrm{ppm} /$ liter $)$ & $\left(\mathrm{t}_{1}\right)$ \\
2 & Sodium nitrate $\mathrm{NaNO}_{3}$ & $(50000 \mathrm{ppm} /$ liter $)$ & $\left(\mathrm{t}_{2}\right)$ \\
3 & Monosodium phosphate $\mathrm{NaH}_{2}\left(\mathrm{PO}_{4}\right)_{3}$ & $(30000 \mathrm{ppm} /$ liter $)$ & $\left(\mathrm{t}_{3}\right)$ \\
\hline
\end{tabular}

Heritabilites are estimated by several methods that use different genetic populations and produced estimates that may vary. Common methods include the variance components method and parent-offspring regression. In this investigation we used the parent- offspring regression as estimate for heritability.

The significance was estimated by $\mathrm{T}$ test by comparison between groups (comparison between mutated plants with unmutated plants).

\section{Results and Discussion}

At the first season of the investigation all mutagenic treatments induced mutants of different desired traits in this crop such as smooth leaves, red and orange petals, earlier flowering and more yielding plants.

Table (1) shows that chosen mutant in M1 generation after applying the mutagen treatments. It is clear from results in Table 1, that mutant differ from the original plants of different safflower genotypes in four main characters i. e. seed yield / plant (S.Y/P), number of days from sowing to flowering (N.D.F), thorns and sleek and petal color. Results show that all treatments (Radiation, Chemicals and Electric shock) have led to mutations in all safflower genotypes. 
Okaz, et al. 2016

Table 1. List of mutants chosen in M1 generation in 2013/2014 season

\begin{tabular}{|c|c|c|c|c|c|c|c|c|}
\hline Genotyp & M.N & S.Y/P & N.D.F & Thorns & Sleel & \multicolumn{2}{|c|}{ Colour flower } \\
\hline & & & & & & Orange & Red & Yellow \\
\hline (L1) & & 26.5 & 130 & $\sqrt{ }$ & & & & $\sqrt{ }$ \\
\hline (L2) & & 15.1 & 131 & $\sqrt{ }$ & & & & $\sqrt{ }$ \\
\hline (L3) & & 8.9 & 130 & $\sqrt{ }$ & & & & $\sqrt{ }$ \\
\hline L1 r1 & 3 & 78.5 & 130 & $\sqrt{ }$ & & $\sqrt{ }$ & & \\
\hline L1 r1 & 4 & 42.7 & 129 & $\sqrt{ }$ & & & & $\sqrt{ }$ \\
\hline L1 r1 & 10 & 56.4 & 130 & $\sqrt{ }$ & & $\sqrt{ }$ & & \\
\hline L1 r1 & 16 & 51.8 & 130 & & & $\sqrt{ }$ & & \\
\hline L1 r2 & 6 & 69.7 & 128 & & & $\sqrt{ }$ & & \\
\hline L1 r2 & 12 & 28.1 & 129 & $\sqrt{ }$ & & $\sqrt{ }$ & & \\
\hline L2 r1 & 1 & 61.5 & 127 & & $\sqrt{ }$ & $\sqrt{ }$ & & \\
\hline L2 r1 & 3 & 57.1 & 128 & & $\sqrt{ }$ & & & $\sqrt{ }$ \\
\hline L2 r1 & 6 & 76.0 & 127 & & $\sqrt{ }$ & $\sqrt{ }$ & & \\
\hline L2 r1 & 7 & 37.4 & 128 & & $\sqrt{ }$ & $\sqrt{ }$ & & \\
\hline L2 r2 & 7 & 29.8 & 129 & & $\sqrt{ }$ & & $\sqrt{ }$ & \\
\hline L2 r2 & 8 & 65.9 & 130 & & $\sqrt{ }$ & $\sqrt{ }$ & & \\
\hline L2 r2 & 9 & 26.5 & 129 & & $\sqrt{ }$ & & & $\sqrt{ }$ \\
\hline L2 r2 & 10 & 20.7 & 129 & & $\sqrt{ }$ & $\sqrt{ }$ & & \\
\hline L3 r1 & 7 & 68.0 & 128 & & $\sqrt{ }$ & $\sqrt{ }$ & & \\
\hline L3 r2 & 1 & 102.8 & 127 & $\sqrt{ }$ & & & & $\sqrt{ }$ \\
\hline L3 r2 & 3 & 99.6 & 128 & $\sqrt{ }$ & & $\sqrt{ }$ & & \\
\hline L1h1 & 4 & 32.3 & 125 & $\sqrt{ }$ & & & & $\sqrt{ }$ \\
\hline L1h1 & 6 & 16.1 & 125 & $\sqrt{ }$ & & & & $\sqrt{ }$ \\
\hline L1h1 & 9 & 59.2 & 126 & $\sqrt{ }$ & & $\sqrt{ }$ & & \\
\hline L1h1 & 10 & 65.2 & 125 & & $\sqrt{ }$ & $\sqrt{ }$ & & \\
\hline L1h1 & 11 & 85.9 & 127 & & $\sqrt{ }$ & $\sqrt{ }$ & & \\
\hline L1h1 & 15 & 28.3 & 125 & & $\sqrt{ }$ & & & $\sqrt{ }$ \\
\hline L1h2 & 6 & 9.9 & 125 & & $\sqrt{ }$ & $\sqrt{ }$ & & \\
\hline L1h2 & 8 & 44.8 & 124 & & $\sqrt{ }$ & & $\sqrt{ }$ & \\
\hline L1h2 & 11 & 19.0 & 124 & $\sqrt{ }$ & & & & $\sqrt{ }$ \\
\hline L1h2 & 20 & 19.1 & 126 & & $\sqrt{ }$ & $\sqrt{ }$ & & \\
\hline L1h3 & 4 & 39.7 & 127 & & $\sqrt{ }$ & $\sqrt{ }$ & & \\
\hline L1h3 & 6 & 34.2 & 128 & & $\sqrt{ }$ & $\sqrt{ }$ & & \\
\hline L1h3 & 8 & 39.9 & 126 & & $\sqrt{ }$ & $\sqrt{ }$ & & \\
\hline L1h3 & 12 & 44.3 & 128 & & $\sqrt{ }$ & & & $\sqrt{ }$ \\
\hline L2h1 & 7 & 70.7 & 127 & & $\sqrt{ }$ & $\sqrt{ }$ & & \\
\hline L2h1 & 8 & 78.9 & 127 & & $\sqrt{ }$ & $\sqrt{ }$ & & \\
\hline L2h1 & 10 & 112.2 & 128 & & $\sqrt{ }$ & & & $\sqrt{ }$ \\
\hline L2h1 & 12 & 132.8 & 128 & $\sqrt{ }$ & & & & $\sqrt{ }$ \\
\hline
\end{tabular}

\begin{tabular}{|c|c|c|c|c|c|c|c|c|}
\hline Genotyp & M.N & S.Y/P & N.D.F & Thorns Sleek & \multicolumn{2}{|c|}{ Colour flower } \\
\hline & & & & & & Orange & Red & Yellow \\
\hline (L1) & & 26.45 & 130 & $\sqrt{ }$ & & & & \\
\hline (L2) & & 15.11 & 131 & $\sqrt{ }$ & & & & \\
\hline (L3) & & 8.89 & 131 & $\sqrt{ }$ & & & & \\
\hline L2h2 & 3 & 97.55 & 129 & $\sqrt{ }$ & & & & $\sqrt{ }$ \\
\hline L2h2 & 4 & 90.6 & 128 & $\sqrt{ }$ & & & & $\sqrt{ }$ \\
\hline L2h2 & 5 & 107.2 & 128 & $\sqrt{ }$ & & & & $\sqrt{ }$ \\
\hline L2h3 & 5 & 122.6 & 128 & & $\sqrt{ }$ & & & $\sqrt{ }$ \\
\hline L2h3 & 13 & 82.39 & 128 & & $\sqrt{ }$ & & & $\sqrt{ }$ \\
\hline L3h1 & 5 & 80.54 & 126 & & $\sqrt{ }$ & $\sqrt{ }$ & & \\
\hline L3h1 & 7 & 77.35 & 125 & & $\sqrt{ }$ & $\sqrt{ }$ & & \\
\hline L3h2 & 5 & 86.55 & 124 & & $\sqrt{ }$ & $\sqrt{ }$ & & \\
\hline L3h2 & 10 & 60.72 & 125 & $\sqrt{ }$ & & & & $\sqrt{ }$ \\
\hline L3h3 & 2 & 177.9 & 128 & & $\sqrt{ }$ & $\sqrt{ }$ & & \\
\hline L3h3 & 5 & 125.3 & 128 & & $\sqrt{ }$ & & $\sqrt{ }$ & \\
\hline L3h3 & 7 & 97.95 & 129 & & $\sqrt{ }$ & & & $\sqrt{ }$ \\
\hline L1t1 & 13 & 106.9 & 126 & & $\sqrt{ }$ & $\sqrt{ }$ & & \\
\hline L1t1 & 9 & 115.3 & 125 & & $\sqrt{ }$ & & $\sqrt{ }$ & \\
\hline L1t2 & 4 & 39.73 & 127 & & $\sqrt{ }$ & & & $\sqrt{ }$ \\
\hline L1t2 & 6 & 23.32 & 125 & & $\sqrt{ }$ & & & $\sqrt{ }$ \\
\hline L1t2 & 7 & 103.7 & 126 & & $\sqrt{ }$ & & & $\sqrt{ }$ \\
\hline L1t2 & 11 & 120.1 & 127 & & $\sqrt{ }$ & & & $\sqrt{ }$ \\
\hline L1t3 & 3 & 104.6 & 126 & $\sqrt{ }$ & & $\sqrt{ }$ & & \\
\hline L2t1 & 5 & 157.7 & 127 & & $\sqrt{ }$ & & & $\sqrt{ }$ \\
\hline L2t1 & 11 & 102.4 & 128 & & $\sqrt{ }$ & & & $\sqrt{ }$ \\
\hline L2t1 & 16 & 153.2 & 127 & & $\sqrt{ }$ & & & $\sqrt{ }$ \\
\hline L2t1 & 20 & 214.2 & 126 & & $\sqrt{ }$ & & & $\sqrt{ }$ \\
\hline L2t2 & 5 & 107 & 125 & $\sqrt{ }$ & & & & $\sqrt{ }$ \\
\hline L2t2 & 13 & 132.6 & 125 & & $\sqrt{ }$ & & & $\sqrt{ }$ \\
\hline L2t2 & 19 & 247.6 & 126 & $\sqrt{ }$ & & & & $\sqrt{ }$ \\
\hline L2t2 & 20 & 307.3 & 126 & $\sqrt{ }$ & & & & $\sqrt{ }$ \\
\hline L2t3 & 2 & 93.42 & 126 & & $\sqrt{ }$ & $\sqrt{ }$ & & \\
\hline L2t3 & 6 & 107.2 & 126 & $\sqrt{ }$ & & & & $\sqrt{ }$ \\
\hline L2t3 & 9 & 77.3 & 127 & $\sqrt{ }$ & & & & $\sqrt{ }$ \\
\hline L2t1 & 1 & 85.8 & 129 & $\sqrt{ }$ & & $\sqrt{ }$ & & \\
\hline L2t1 & 5 & 57.13 & 129 & $\sqrt{ }$ & & & & $\sqrt{ }$ \\
\hline L2t2 & 4 & 97.54 & 128 & & $\sqrt{ }$ & & & $\sqrt{ }$ \\
\hline L2t3 & 1 & 140 & 126 & & $\sqrt{ }$ & $\sqrt{ }$ & & \\
\hline L2t3 & 3 & 102 & 127 & $\sqrt{ }$ & & & & $\sqrt{ }$ \\
\hline
\end{tabular}


Obtained plants in $\mathrm{M}_{1}$ which shows in Table 1 were planted to get a second and third generation. The number of plants which maintain the mutations in $\mathrm{M}_{2}$ and $\mathrm{M}_{3}$ are shown in Table 2.

Table 2. Number of plants which have mutation in different generation

\begin{tabular}{|c|c|c|c|c|c|c|c|c|c|c|c|}
\hline \multicolumn{5}{|c|}{ Radiation } & \multicolumn{5}{|c|}{ chemical } & \multicolumn{4}{|c|}{ electric shock } \\
\hline & M1 & M2 & M3 & & M1 & M2 & M3 & & M1 & M2 & M3 \\
\hline L1r1 & 22 & 8 & 4 & L1h1 & 7 & 8 & 6 & L1t1 & 5 & 4 & 2 \\
\hline L1r2 & 20 & 4 & 2 & L1h2 & 11 & 8 & 4 & L1t2 & 5 & 8 & 4 \\
\hline L2r1 & 15 & 8 & 4 & L1h3 & 8 & 6 & 4 & L1t3 & 5 & 3 & 1 \\
\hline L2r2 & 14 & 8 & 4 & L2h1 & 19 & 8 & 4 & L2t1 & 18 & 6 & 4 \\
\hline L3r1 & 14 & 4 & 1 & L2h2 & 20 & 8 & 3 & L2t2 & 13 & 7 & 4 \\
\hline L3r2 & 16 & 4 & 2 & L2h3 & 3 & 6 & 2 & L2t3 & 7 & 7 & 3 \\
\hline & & & & L3h1 & 21 & 5 & 2 & L3t1 & 10 & 6 & 2 \\
\hline & & & & L3h2 & 20 & 6 & 2 & L3t2 & 8 & 4 & 1 \\
\hline & & & & L3h3 & 14 & 5 & 3 & L3t3 & 10 & 6 & 2 \\
\hline
\end{tabular}

Results in Table (2) shows that the numbers of plants which maintain of mutations until the third generation were 70 plants.

The means and variances of the mutants which cached from all mutagenic treatment were calculated and compared with that of the same number of plants representing control treatment for the two main traits i.e. seed yield/plant and number of days from sowing to flowering (Table 3).

\section{Effect of Gamma rays:}

Data in Table (3) and Fig. 1 and 2 shows that Gamma rays led to obtain early plants in flowering. Line No.2 was more response to treatment of gamma rays than another genotypes in flowering date. $\mathrm{L}_{2} \mathrm{r}_{1}$ gave the earliest (127.5 day) plants its early 5 days compared with untreated plants $\mathrm{L}_{2}$ (132.67 day). In general, treatment $\mathrm{r}_{1}$ was more effective than another to induce mutation and gave mutant with early flowering.

All plants which maintain the mutations until M3 were surpassed untreated plants in seed yield. Line No. 3 was most responsive to radiation and plants of $L_{3} r_{2}$ and $L_{3} r_{1}$ were given 112.13 and $105.01 \mathrm{~g}$ of seed yield, respectively compared with $40.28 \mathrm{~g}$ obtained from untreated plants. So, the increasing percentage from untreated plants was 179.04 and $161.67 \%$, respectively.

Line No.2 occupied the second place about the responsive to radiation. $\mathrm{L}_{2} \mathrm{r}_{2}$ and $\mathrm{L}_{2} \mathrm{r}_{1}$ gave 89.96 and $74.30 \mathrm{~g}$, respectively, compared with $31.51 \mathrm{~g}$ obtained from untreated plants. Mutants $\mathrm{L}_{2} \mathrm{r}_{2}$ and $\mathrm{L}_{2} \mathrm{r}_{1}$ surpassed original plants in seed yield /plant with 185.49 and $135.49 \%$, respectively. 
Table 3. Means and variances for safflower genotypes under different treatments of mutagenic through generation.

\begin{tabular}{|c|c|c|c|c|c|c|c|c|c|c|c|c|c|}
\hline \multicolumn{14}{|c|}{ Radiation } \\
\hline \multirow{3}{*}{ Lines characters } & \multirow{3}{*}{ Season } & \multicolumn{6}{|c|}{ N.D.F } & \multicolumn{6}{|c|}{ S.Y /P } \\
\hline & & \multicolumn{3}{|c|}{ means $\pm S . E$} & \multicolumn{3}{|c|}{ variance } & \multicolumn{3}{|c|}{ means $\pm S . E$} & \multicolumn{3}{|c|}{ variance } \\
\hline & & M1 & M2 & M3 & M1 & M2 & M3 & M1 & $\mathrm{M} 2$ & M3 & M1 & M2 & M3 \\
\hline \multirow{3}{*}{ L1 } & r1 & $130.44 \pm 0.12$ & $127.75 \pm 0.31$ & $127.21 \pm 1.06^{*}$ & $0.26 *$ & $0.79^{*}$ & $8.98^{*}$ & $66.69 \pm 6.04^{*}$ & $34.43 \pm 2.73^{*}$ & * $71.05 \pm 6.48^{*}$ & $655.95^{*}$ & $\begin{array}{ll}* & 59.41^{*}\end{array}$ & \begin{tabular}{l|l}
$*$ & 1006.49 \\
\end{tabular} \\
\hline & $\mathrm{r} 2$ & $128.23 \pm 0.12^{*}$ & * $128.25 \pm 0.48$ & $128.42 \pm 0.71$ & $0.19^{*}$ & $0.92^{*}$ & $2.00^{*}$ & $80.31 \pm 10.18^{*}$ & * $23.32 \pm 5.46$ & $93.07 \pm 9.61^{*}$ & 1517.37 & **119.05* & ${ }^{*} 1107.71$ \\
\hline & Cont. & $130.33 \pm 0.33$ & $129.6 \pm 0.24$ & $130.67 \pm 0.33$ & 0.33 & 0.3 & 0.33 & $26.45 \pm 0.39$ & $25.7 \pm 0.21$ & $62.87 \pm 0.39$ & 0.6 & 0.18 & 0.6 \\
\hline \multirow{3}{*}{12} & $\mathrm{r} 1$ & $127.43 \pm 0.20^{*}$ & * $126.88 \pm 0.44$ & $127.54 \pm 0.51^{*}$ & $0.29 *$ & $1.55^{*}$ & $1.81^{*}$ & $70.52 \pm 7.67^{*}$ & $31.55 \pm 7.11^{*}$ & * $74.3 \pm 9.98^{*}$ & 411.788 & $4=404.32^{*}$ & ${ }^{*} 2389.88^{*}$ \\
\hline & $\mathrm{r} 2$ & $129.3 \pm 0.15$ & $130 \pm 0.38$ & $130.21 \pm 0.56$ & $0.23^{*}$ & $1.14^{*}$ & $2.50^{*}$ & $60.24 \pm 8.83^{*}$ & $51.64 \pm 3.95^{*}$ & * $89.96 \pm 9.96^{*}$ & $779.55^{*}$ & $\begin{array}{ll}* & 124.53^{*} \\
\end{array}$ & $2379.82^{*}$ \\
\hline & Cont. & $130.67 \pm 0.33$ & $132.25 \pm 0.48$ & $132.67 \pm 0.67$ & 0.33 & 0.92 & 1.33 & $15.11 \pm 0.42$ & $12.12 \pm 0.70$ & $31.51 \pm 0.42$ & 0.71 & 1.97 & 0.71 \\
\hline \multirow{3}{*}{13} & $\mathrm{r} 1$ & $128.88 \pm 0.13$ & $129.5 \pm 0.29$ & $127 \pm 0.89^{*}$ & 0.125 & $0.33^{*}$ & $4.00^{*}$ & $109.53 \pm 17.54$ & $25.06 \pm 2.73^{*}$ & $112.13 \pm 10.10$ & 2461.69 & $29.84^{*}$ & 1223.15 \\
\hline & $r 2$ & $127.6 \pm 0.04^{*}$ & $128.5 \pm 0.29 *$ & $128.25 \pm 0.48$ & 0.02 & $0.33^{*}$ & $0.92^{*}$ & $81.76 \pm 6.8^{*}$ & $46.61 \pm 3.18^{*}$ & $105.01 \pm 14.60$ & $* 461.99^{*}$ & * $40.32^{*}$ & * $2345.83^{3}$ \\
\hline & Cont. & $130.25 \pm 0.48$ & $130.5 \pm 0.50$ & $130.33 \pm 0.33$ & 0.92 & 0.5 & 0.33 & $8.89 \pm 0.32$ & $8.25 \pm 0.50$ & $40.28 \pm 0.32$ & 0.4 & 0.51 & 0.4 \\
\hline \multicolumn{14}{|c|}{ Chemical } \\
\hline \multirow{3}{*}{ Lines } & & \multicolumn{6}{|c|}{ N.D.F } & \multicolumn{6}{|c|}{$S . Y / P$} \\
\hline & \multirow{2}{*}{ Seasons } & \multicolumn{3}{|c|}{ means $\pm S . E$} & \multicolumn{3}{|c|}{ variance } & \multicolumn{3}{|c|}{ means $\pm S . E$} & & variance & \\
\hline & & M1 & M2 & M3 & M1 & M2 & M3 & M1 & M2 & M3 & M1 & M2 & M3 \\
\hline & h1 & $125.73 \pm 0.15^{*}$ & $=122 \pm 0.57^{*}$ & $116.83 \pm 0.40^{*}$ & 0.49 & $0.57^{*}$ & $1.27^{*}$ & $115.51+8.65 *$ & $17.8 \pm 2.90$ & $93.2 \pm 12.54^{*}$ & 1644.35 & $67.17^{*}$ & $3299.83^{\circ}$ \\
\hline 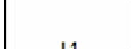 & h2 & $124.25 \pm 0.14^{*}$ & $121.38 \pm 0.18$ & $120.92 \pm 0.38$ & $0.41^{*}$ & $0.27^{*}$ & $1.14^{*}$ & $43.41 \pm 6.74^{*}$ & $24.64 \pm 1$ & $127.17 \pm 8.28$ & "908.49* & * $7.93^{*}$ & 1645.95 \\
\hline 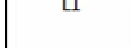 & h3 & $127.93 \pm 0.18$ & $125.5 \pm 0.22$ & $125.5 \pm 0.48^{*}$ & $0.50^{*}$ & $0.30^{*}$ & $1.37^{*}$ & $64.41 \pm 7.39 *$ & $23.13 \pm 3.78$ & $90.8 \pm 8.39^{*}$ & $820.05 *$ & 85.54 & 1055.14 \\
\hline & Cont. & $130.33 \pm 0.33$ & $129.6 \pm 0.24$ & $130.67 \pm 0.33$ & 0.33 & 0.3 & 0.33 & $26.45 \pm 0.39$ & $25.7 \pm 0.21$ & $62.87 \pm 0.39$ & 0.6 & 0.18 & 0.6 \\
\hline & h1 & $127.43 \pm 0.20^{*}$ & $121.38 \pm 0.18$ & $=127.33 \pm 0.31$ & $0.57^{*}$ & 0.27 & 0.79 & $101.28 \pm 10.80$ & $27.72 \pm 1.38$ & $=53.51 \pm 4.09 *$ & 1634.32 & 15.25 & 385.25 \\
\hline 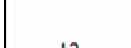 & h2 & $128.36 \pm 0.20$ & $125.5 \pm 0.33^{*}$ & $130 \pm 0.38$ & $0.55 *$ & $0.86^{*}$ & 1.14 & $128.06 \pm 12.87$ & $36.67 \pm 2.74$ & $=47.67 \pm 3.96^{*}$ & 2318.34 & $60.03 *$ & * $375.72^{*}$ \\
\hline L & h3 & $128.19 \pm 0.16$ & $130.5 \pm 0.22$ & $128.56 \pm 0.22$ & $0.43^{*}$ & 0.3 & 0.3 & $118.65 \pm 13.61$ & $24.03+5.43^{2}$ & $58.2 \pm 6.67^{*}$ & 2961.72 & 176.68 & 800.69 \\
\hline & Cont. & $130.67 \pm 0.33$ & $132.25 \pm 0.48$ & $132.67 \pm 0.67$ & 0.33 & 0.92 & 1.33 & $15.11 \pm 0.42$ & $12.12 \pm 0.70$ & $31.51 \pm 0.42$ & 0.71 & 1.97 & 0.71 \\
\hline & h1 & $125.71 \pm 0.29 *$ & $=129.8 \pm 0.49$ & $126.83 \pm 0.56 *$ & 0.57 & $1.20^{*}$ & $1.87^{*}$ & $83.12 \pm 6.37^{*}$ & $6.84 \pm 12.99$ & $97.29 \pm 9.66^{*}$ & $283.71 *$ & 844.32 & $1679.81^{*}$ \\
\hline ב & h2 & $124.27 \pm 0.27$ & $128 \pm 0.26$ & $125.5 \pm 0.67^{*}$ & 0.82 & $0.40^{*}$ & $2.67^{*}$ & $102.05 \pm 11.07$ & $37.16 \pm 4.72$ & $=73.52 \pm 6.89 *$ & 1347.04 & 133.84 & $* 854.81^{*}$ \\
\hline$[s$ & h3 & $127.88 \pm 0.23$ & $132.6 \pm 0.24$ & $128 \pm 0.49$ & 0.41 & $0.30^{*}$ & $1.20^{*}$ & $140.23 \pm 18.16$ & $30.91 \pm 4.43$ & $97.32 \pm 6.65 *$ & 2638.70 & $98.29 \%$ & $530.82^{*}$ \\
\hline & Cont. & $130.25 \pm 0.48$ & $130.5 \pm 0.50$ & $130.33 \pm 0.33$ & 0.92 & 0.5 & 0.33 & $8.89 \pm 0.32$ & $8.25 \pm 0.50$ & $40.28 \pm 0.32$ & 0.4 & 0.51 & 0.4 \\
\hline & & & & & & lectric s & hock & & & & & & \\
\hline characters & & & & N.D.F & & & & & & $S . Y / P$ & & & \\
\hline & Seasons & & means $\pm S . E$ & & & varianc & & & means $\pm S . E$ & & & variance & \\
\hline & & M1 & M2 & M3 & M1 & M2 & M3 & M1 & M2 & M3 & M1 & M2 & M3 \\
\hline & $\mathrm{t} 1$ & $125.21 \pm 0.21^{*}$ & $124.5 \pm 0.19$ & $127 \pm 0.48^{*}$ & $0.84^{*}$ & $0.14^{*}$ & $0.92^{*}$ & $136.17 \pm 14.40$ & $=9.55 \pm 1.08$ & $94.21 \pm 19.13^{*}$ & 3942.30 & $4.63^{*}$ & 3292.67 \\
\hline 1 & $\mathrm{t} 2$ & $127.20 \pm 0.20^{*}$ & $126.88 \pm 0.23$ & $127.21 \pm 0.33^{*}$ & $0.8^{*}$ & $0.41^{*}$ & $0.86^{*}$ & $88.02+9.14^{*}$ & $38.07 \pm 6.39 *$ & $100.05+9.16^{*}$ & $\begin{array}{ll}* 1672.35 \\
\end{array}$ & $327.10^{*}$ & \begin{tabular}{|l|l|}
$*$ & $1929,43^{\prime}$
\end{tabular} \\
\hline$u$ & $\mathrm{t} 3$ & $126.67 \pm 0.33^{*}$ & $125.67 \pm 0.33$ & $126.33 \pm 0.33^{*}$ & $0.33^{*}$ & $0.33^{*}$ & $0.33^{*}$ & $65.63 \pm 29.64^{*}$ & $16.41 \pm 4.34$ & $90.4 \pm 12.03^{*}$ & 2635.70 & $56.63^{*}$ & $=1302.10^{\circ}$ \\
\hline & Cont. & $130.33 \pm 0.33$ & $129.6 \pm 0.24$ & $130.67 \pm 0.33$ & 0.33 & 0.3 & 0.33 & $26.45 \pm 0.39$ & $25.7 \pm 0.21$ & $62.87 \pm 0.39$ & 0.6 & 0.18 & 0.6 \\
\hline & $\mathrm{t} 1$ & $127.81 \pm 0.16$ & $125.83 \pm 0.31$ & $125.5 \pm 0.37^{*}$ & $0.56^{*}$ & 0.57 & 0.8 & $155.25 \pm 10.39$ & $20.44 \pm 1.41^{2}$ & $=104.64 \pm 6.40^{*}$ & 2635.70 & $11.88^{*}$ & $\begin{array}{l}* 738.38^{*} \\
\end{array}$ \\
\hline 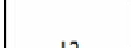 & $\mathrm{t} 2$ & $125.2 \pm 0.14^{*}$ & $127 \pm 0.31^{*}$ & $127.05 \pm 0.22^{*}$ & $0.38^{*}$ & 0.67 & 0.33 & $180.68 \pm 20.51$ & $25.88+5.65^{*}$ & $75.4 \pm 7.48^{*}$ & 8413.09 & $223.1^{*}$ & 1119.04 \\
\hline 2 & t3 & $126.14 \pm 0.25^{*}$ & $127.14 \pm 0.34$ & $127.33 \pm 0.22^{*}$ & $0.9 *$ & 0.81 & 0.3 & $115.7 \pm 6.82^{*}$ & $15.67 \pm 1.79$ & $128 \pm 10.63^{*}$ & $650.23^{*}$ & $\begin{array}{l} \\
\end{array} 22.38^{*}$ & 2373.69 \\
\hline & Cont. & $130.67 \pm 0.33$ & $132.25 \pm 0.48$ & $132.67 \pm 0.67$ & 0.33 & 0.92 & 1.33 & $15.11 \pm 0.42$ & $12.12 \pm 0.70$ & $31.51 \pm 0.42$ & 0.71 & 1.97 & 0.71 \\
\hline & $\mathrm{t} 1$ & $129.2 \pm 0.37$ & $128 \pm 0.37$ & $128.5 \pm 0.31$ & 0.7 & $0.8^{*}$ & $0.57^{*}$ & $76.94 \pm 6025^{*}$ & $50.5 \pm 5.31^{*}$ & $102.96 \pm 7.13^{*}$ & $\begin{array}{l}* 650.23^{*} \\
\end{array}$ & * $169.19 *$ & * $914.13^{*}$ \\
\hline 1 & $\mathrm{t} 2$ & $128.4 \pm 0.51$ & $129.75 \pm 0.48$ & $130 \pm 0.29$ & $1.3^{*}$ & $0.92^{*}$ & $0.33^{*}$ & $113.17 \pm 12.27$ & $\sqrt{5.31 \pm 12.37}$ & $100.92+9.63^{*}$ & $\begin{array}{ll}* & 753.34^{*}\end{array}$ & ${ }^{*} 612.20^{*}$ & $*^{*} 1112.72^{*}$ \\
\hline T3 & $\mathrm{t} 3$ & $126.2 \pm 0.20^{*}$ & $126.67 \pm 0.49$ & $127 \pm 0.83^{*}$ & 0.2 & $1.47^{*}$ & $4.17^{*}$ & $122.63 \pm 7.70^{*}$ & $27.88 \pm 1.22^{*}$ & * $49.42 \pm 2.70^{*}$ & $296.12^{*}$ & \begin{tabular}{|l|l|}
$*$ & $8.93^{*}$ \\
\end{tabular} & $130.74^{*}$ \\
\hline & Cont. & $130.25 \pm 0.48$ & $130.5 \pm 0.50$ & $130.33 \pm 0.33$ & 0.92 & 0.5 & 0.33 & $8.89 \pm 0.32$ & $8.25 \pm 0.50$ & $40.28 \pm 0.32$ & 0.4 & 0.51 & 0.4 \\
\hline
\end{tabular}


In spite of Line No.1 gave the highest seed yield $(62.87 \mathrm{~g})$, but it was taken the third place about responsive to radiation treatments. $\mathrm{L}_{1} \mathrm{r}_{2}$ and $\mathrm{L}_{1} \mathrm{r}_{1}$ gave 93.07 and $71.05 \mathrm{~g}$, respectively, compared with $62.87 \mathrm{~g}$ obtained from untreated plants. Mutants $\mathrm{L}_{1} \mathrm{r}_{2}$ and $\mathrm{L}_{1} \mathrm{r}_{1}$ surpassed original plants in seed yield /plant with 48.04 and $13.01 \%$, respectively.
Results obtained from effect of radiation illustrate that; treatment $r_{2}$ was more effective than another to induce mutation and gave mutant with high yielding. The results agreed with those of Mia and Shaikh (1997) and Sheeba et al. (2005).

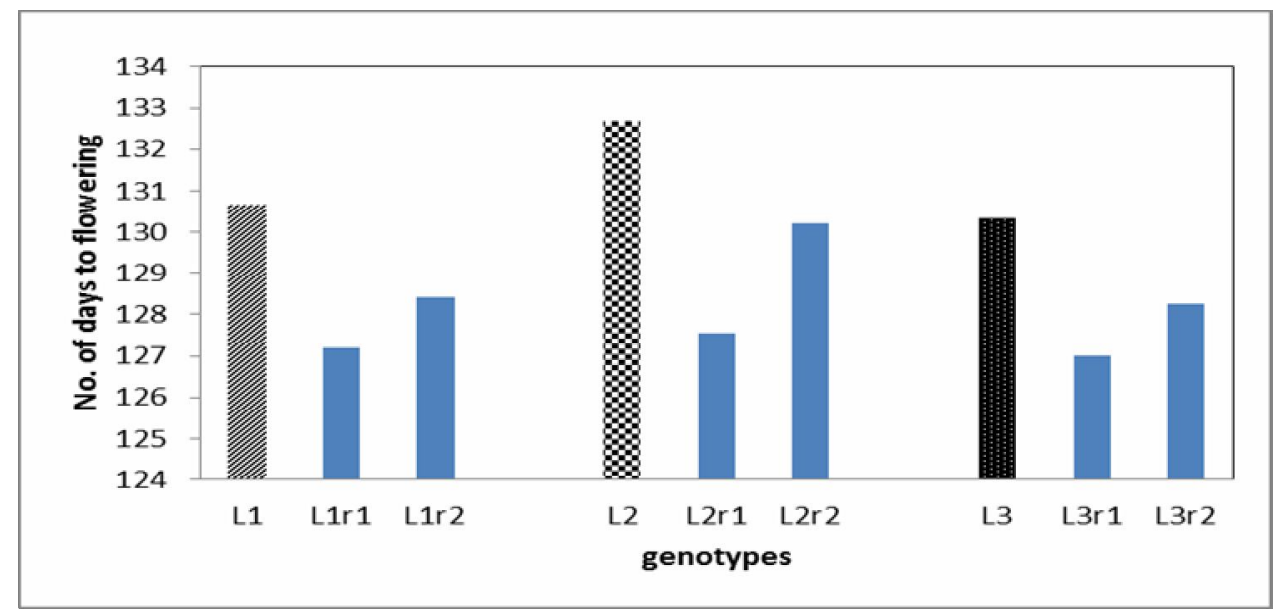

Fig.1: Number of days to flowering of safflower genotypes under different gamma rays treatments

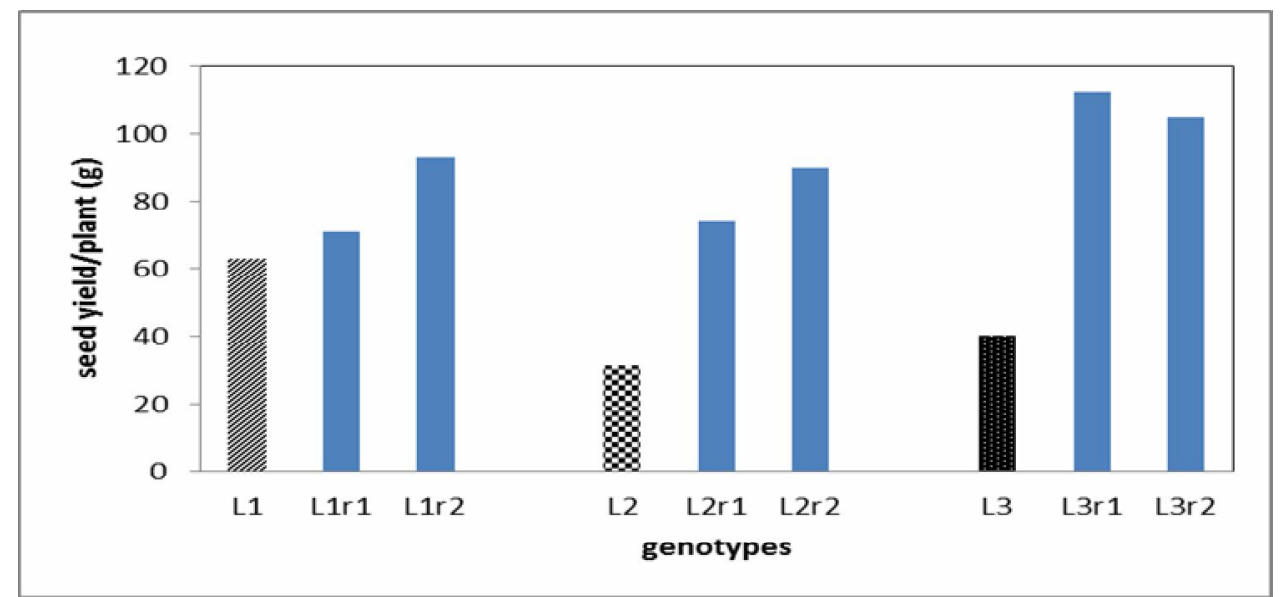

Fig.2: Seed yield/ plant of safflower genotypes under different gamma rays treatments 
The parent-offspring regression coefficients values (Table 4) represent heritability in narrow sense reached 0.51 and 0.68 for N.D.F and 0.19 and 0.02 for S.Y/plant of $\mathrm{M}_{2}$ and $\mathrm{M}_{3}$ generation respectively.

\section{Effect of Chemical treatments:}

Results in Table (3) and Fig. 3 and 4 illustrated that Line No.1 was more response to chemicals treatment about flowering than another genotypes and its gave early flowering plants. $\mathrm{L}_{1} \mathrm{~h}_{1}, \mathrm{~L}_{1} \mathrm{~h}_{2}$ and $\mathrm{L}_{1} \mathrm{~h}_{3}$ gave the earliest (113.83,120.92 and 125.5 day, respectively) plants its earlier 13.84, 9.75 and 5.17 days, respectively, than untreated plants $\mathrm{L}_{1}(132$ .67 day). In general, treatment $r_{1}$ was more effective than another to induce mutation and gave mutant with early flowering.
All plants which maintain the mutations until M3 were surpassed untreated plants in seed yield. The highest seed yield /plant (127.17 g) was obtained from $\mathrm{L}_{1} \mathrm{~h}_{2}$, but untreated plant $\mathrm{L}_{2}$ gave $62.87 \mathrm{~g}$. So, L1h2 surpassed untreated plants with $102.27 \%$ for seed yield / plant.

Line No.3 occupied the second place in seed yield/plant. Where, both of $\mathrm{L}_{3} \mathrm{~h}_{1}$ and $\mathrm{L}_{3} \mathrm{~h}_{2}$ gave $97.3 \mathrm{~g}$. This means that $\mathrm{L}_{3} \mathrm{~h}_{1}$ and $\mathrm{L}_{3} \mathrm{~h}_{2}$ increased $142.5 \%$ in seed yield/plant more than $\mathrm{L}_{3}$ which gave $40.3 \mathrm{~g}$. This result coincides with Dhole et al. (2003).

The parent-offspring regression coefficients values (Table 5) represent heritability in narrow sense reached 0.72 and 0.89 for N.D.F and 0.10 and -1.07 for $\mathrm{S}$.Y/plant of $\mathrm{M}_{2}$ and $\mathrm{M}_{3}$ generation respectively.

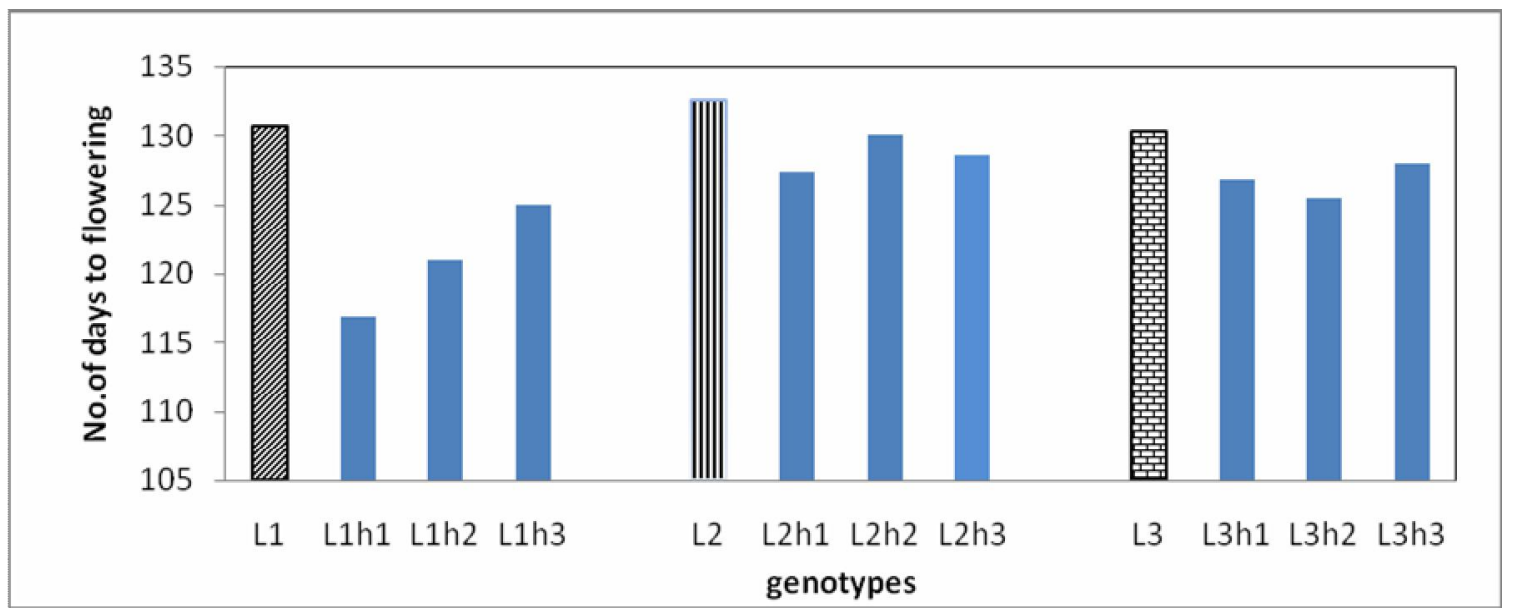

Fig.3: Number of days to flowering of safflower genotypes under different chemical treatments 


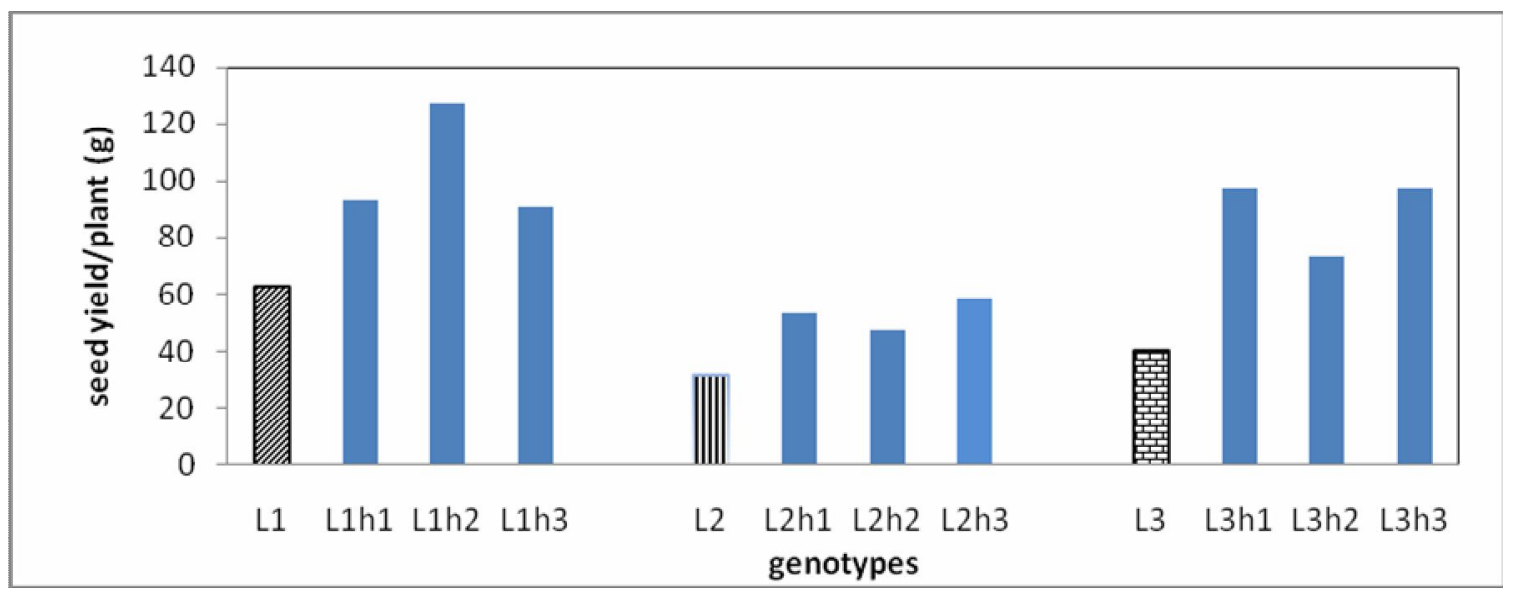

Fig.4: Seed yield/ plant of safflower genotypes under different chemical treatments

\section{Effect of Electric shock:}

Using of electric shock caused to obtain early plants in flowering from all genotypes. Results in Table (3) and Fig. 5 and 6 revealed that the earliest plants were obtained from $\mathrm{L}_{2} \mathrm{t}_{1}$ (125.5 day). $\mathrm{L}_{2} \mathrm{t}_{1}$ was earlier 12.53 days than untreated plants $\mathrm{L}_{2}$ (132.67 day).

All plants which maintain the mutations until M3 were surpassed untreated plants in seed yield. Line No. 2 was most responsive to electric shock and gave plants with high seed yield/plant. Average of seed from treated plants was 95, 102.7 and 84.7 from treated plants of $\mathrm{L}_{1}, \mathrm{~L}_{2}$ and $\mathrm{L}_{3}$, respectively. The highest seed yield (128g) was obtained from $\mathrm{L}_{2} \mathrm{t}_{3}$ with percentage of increasing 306.22 $\%$ from seed yield of untreated plants $(31.51 \mathrm{~g})$. This result coincides with (Ahmad 2011) when used electric shock on wheat.

The parent-offspring regression coefficients values (Table 6) represent heritability in narrow sense reached 0.59 and 0.70 for N.D.F and 0.10 and -0.49 for $\mathrm{S} . \mathrm{Y} /$ plant of $\mathrm{M}_{2}$ and $\mathrm{M}_{3}$ generation respectively.

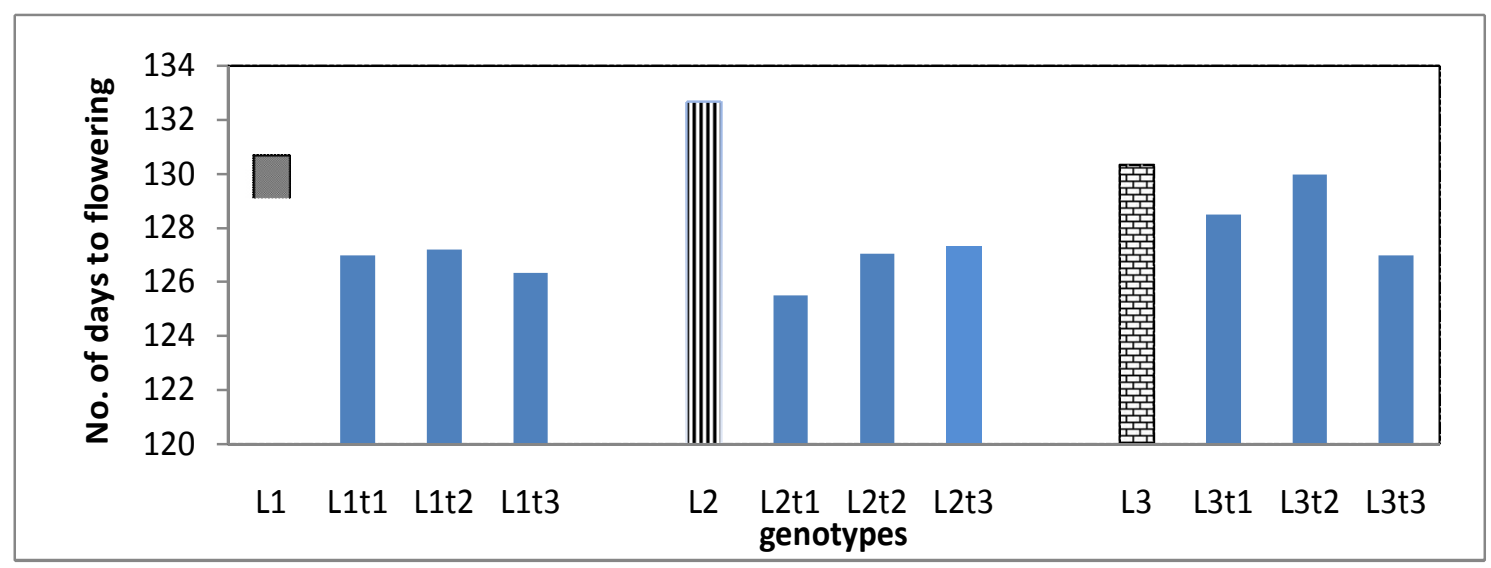

Fig.5: Number of days to flowering of safflower genotypes under different electric shock treatments 


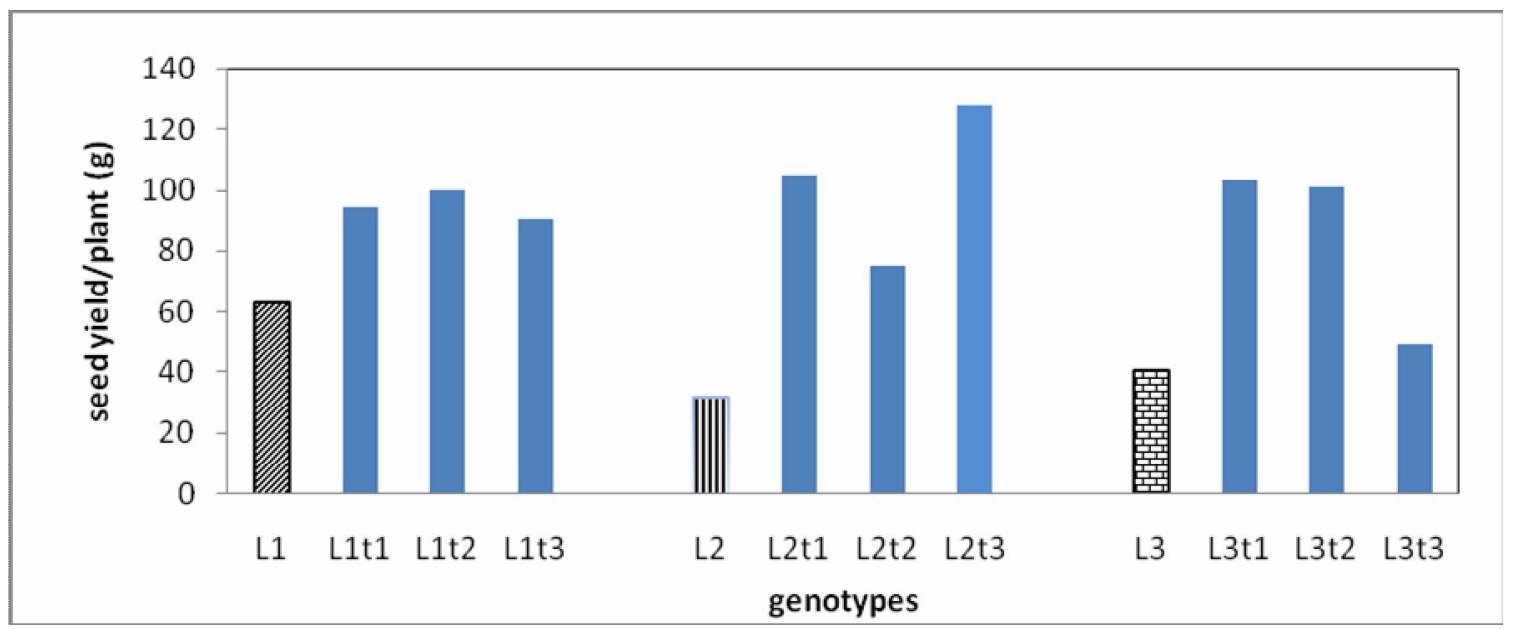

Fig.6: Seed yield/plant of safflower genotypes under different electric shock treatments

Table 4. The morphological variation and parent-offspring regression in mutated plants derived from gamma rays treatments

\begin{tabular}{|l|c|c|c|c|c|c|c|}
\hline \multirow{2}{*}{$\begin{array}{c}\text { Characters } \\
\text { genotype }\end{array}$} & \multicolumn{3}{|c|}{ N.D.F.F } & \multicolumn{3}{c|}{ S.Y/P } & M1 \\
\cline { 2 - 7 } & M2 & M3 & M1 & M2 & M3 & color flower- Texture plant \\
\hline line 32 (L1) & 130.33 & 130.55 & 130.67 & 26.45 & 25.70 & 62.87 & thorns - yellow \\
\hline line 37 (L2) & 131.23 & 132.25 & 132.67 & 15.11 & 12.12 & 31.51 & thorns - yellow \\
\hline line40 (L3) & 130.9 & 131 & 130.33 & 8.89 & 8.25 & 40.28 & thorns - yellow \\
\hline L1 r1-3 & 130 & 127 & 127 & 78.48 & 49.73 & 84.77 & thorns - orange \\
\hline L1 r1-4 & 129 & 127 & 128 & 42.70 & 63.16 & 111.42 & thorns - yellow \\
\hline L1 r1-10 & 130 & 127 & 128 & 56.37 & 67.28 & 117.33 & thorns - orange \\
\hline L1 r1-16 & 130 & 128 & 127 & 51.84 & 47.33 & 53.12 & sleek -orange \\
\hline L1 r2-6 & 128 & 128 & 128 & 69.74 & 61.70 & 102.45 & sleek -orange \\
\hline L1 r2-12 & 129 & 128 & 128 & 28.12 & 36.27 & 78.41 & thorns - orange \\
\hline L2 r1-1 & 127 & 126 & 127 & 61.51 & 39.79 & 50.52 & sleek -orange \\
\hline L2 r1-3 & 128 & 126 & 128 & 57.13 & 89.42 & 96.50 & sleek - yellow \\
\hline L2 r1-6 & 127 & 126 & 127 & 75.99 & 44.17 & 75.55 & sleek -orange \\
\hline L2 r1-7 & 128 & 127 & 127 & 37.39 & 44.34 & 67.63 & sleek -orange \\
\hline L2 r2-7 & 129 & 130 & 130 & 29.79 & 81.52 & 100.56 & sleek - red \\
\hline L2 r2-8 & 130 & 130 & 129 & 65.90 & 62.87 & 60.12 & sleek -orange \\
\hline L2 r2-9 & 129 & 129 & 130 & 26.47 & 56.51 & 150.12 & sleek - yellow \\
\hline L2 r2-10 & 129 & 130 & 130 & 20.74 & 74.70 & 75.17 & sleek -orange \\
\hline L3 r1-7 & 128 & 129 & 130 & 67.99 & 30.65 & 137.99 & sleek -orange \\
\hline L3 r2-1 & 127 & 128 & 128 & 102.75 & 47.11 & 190.45 & thorns - yellow \\
\hline L3 r2-3 & 128 & 128 & 127 & 99.63 & 51.62 & 99.74 & thorns - orange \\
\hline regression coefficients & 0.51 & 0.68 & & -0.19 & 0.02 & \\
\hline
\end{tabular}


Table 5. The morphological variations and parent-offspring regression in mutated plants derived from chemicals treatments

\begin{tabular}{|c|c|c|c|c|c|c|c|}
\hline \multirow{2}{*}{$\begin{array}{l}\text { Characters } \\
\text { genotype }\end{array}$} & \multicolumn{3}{|c|}{ N.D.F.F } & \multicolumn{3}{|c|}{$\mathbf{S . Y} / \mathbf{P}$} & \multirow[b]{2}{*}{ color flower- Texture plant } \\
\hline & M1 & M2 & M3 & M1 & M2 & M3 & \\
\hline line $32(\mathrm{~L} 1)$ & 130.25 & 130.57 & 130.67 & 26.45 & 16.7 & 62.87 & thorns -yellow \\
\hline line 37 (L2) & 131.34 & 126.84 & 132.67 & 15.11 & 12.12 & 31.51 & thorns- yellow \\
\hline line40 (L3) & 130.25 & 126.53 & 130.33 & 8.89 & 8.25 & 40.28 & thorns - yellow \\
\hline L1h1-4 & 125 & 121 & 117 & 32.28 & 22.80 & 157.21 & sleek - yellow \\
\hline L1h1-6 & 125 & 121 & 116 & 16.11 & 28.23 & 139.51 & sleek - yellow \\
\hline L1h1-9 & 126 & 120 & 116 & 59.15 & 39.95 & 83.78 & sleek- orange \\
\hline L1h1-10 & 125 & 123 & 116 & 65.16 & 26.03 & 93.99 & sleek- orange \\
\hline L1h1-11 & 127 & 121 & 117 & 85.87 & 31.50 & 56.51 & sleek -orange \\
\hline L1h1-15 & 125 & 122 & 116 & 28.29 & 16.60 & 247.33 & thorns- yellow \\
\hline L1h2-6 & 125 & 121 & 120 & 9.90 & 29.87 & 91.21 & sleek -orange \\
\hline L1h2-8 & 124 & 121 & 120 & 44.79 & 34.91 & 119.87 & sleek - red \\
\hline L1h2-11 & 124 & 123 & 121 & 19.04 & 32.84 & 124.73 & sleek - yellow \\
\hline L1h2-20 & 126 & 121 & 122 & 19.05 & 27.57 & 168.34 & sleek -orange \\
\hline L1h3-4 & 127 & 125 & 126 & 39.68 & 35.95 & 167.64 & sleek -orange \\
\hline L1h3-6 & 128 & 126 & 126 & 34.20 & 50.48 & 89.54 & sleek -orange \\
\hline L1h3-8 & 126 & 126 & 125 & 39.85 & 50.12 & 125.33 & sleek -orange \\
\hline L1h3-12 & 128 & 125 & 125 & 44.26 & 34.29 & 66.29 & sleek - yellow \\
\hline L2h1-7 & 127 & 122 & 127 & 70.66 & 43.26 & 80.33 & thorns -orange \\
\hline L2h1-8 & 127 & 121 & 128 & 78.89 & 34.48 & 63.48 & thorns - orange \\
\hline L2h1-10 & 128 & 122 & 128 & 112.24 & 30.93 & 105.05 & thorns - yellow \\
\hline L2h1-12 & 128 & 121 & 127 & 132.75 & 39.53 & 32.27 & thorns- yellow \\
\hline L2h2-3 & 129 & 126 & 130 & 97.55 & 59.93 & 62.69 & thorns -yellow \\
\hline L2h2-4 & 128 & 125 & 129 & 90.60 & 51.83 & 95.72 & thorns- yellow \\
\hline L2h2-5 & 128 & 125 & 129 & 107.21 & 45.72 & 73.16 & thorns - yellow \\
\hline L2h3-5 & 128 & 130 & 128 & 122.56 & 47.36 & 65.74 & sleek - yellow \\
\hline L2h3-13 & 128 & 129 & 129 & 82.39 & 29.74 & 120.46 & sleek - yellow \\
\hline L3h1-5 & 126 & 129 & 127 & 80.54 & 91.25 & 133.85 & sleek-orange \\
\hline L3h1-7 & 125 & 129 & 126 & 77.35 & 28.58 & 203.31 & sleek- orange \\
\hline L3h2-5 & 124 & 128 & 125 & 86.55 & 43.63 & 90.35 & sleek -orange \\
\hline L3h2-10 & 125 & 128 & 126 & 60.72 & 41.94 & 134.50 & thorns- yellow \\
\hline L3h3-2 & 128 & 130 & 128 & 177.9 & 31.50 & 138.89 & sleek -orange \\
\hline L3h3-5 & 128 & 130 & 129 & 125.28 & 51.58 & 103.88 & sleek - red \\
\hline L3h3-7 & 129 & 129 & 128 & 97.95 & 47.65 & 109.77 & sleek - yellow \\
\hline \multicolumn{2}{|c|}{ regression coefficients } & 0.72 & 0.89 & & 0.10 & -1.07 & \\
\hline
\end{tabular}


Table 6. The morphological variations and parent-offspring regression in mutated plants derived from electric shock treatments

\begin{tabular}{|c|c|c|c|c|c|c|c|}
\hline \multirow{2}{*}{ Characters } & \multicolumn{3}{|c|}{ N.D.F.F } & \multicolumn{3}{|c|}{ S.Y/P } & \\
\cline { 2 - 8 } genotype & M1 & M2 & M3 & M1 & M2 & M3 & color flower- Texture plant \\
\hline line 32 (L1) & 130.19 & 129.39 & 130.67 & 26.45 & 16.70 & 62.67 & thorns - yellow \\
\hline line 37 (L2) & 131.23 & 132.43 & 132.67 & 15.11 & 12.12 & 31.51 & thorns- yellow \\
\hline line40 (L3) & 130.15 & 130.55 & 130.33 & 8.89 & 8.25 & 40.28 & thorns- yellow \\
\hline L1t1-13 & 126 & 125 & 127 & 106.90 & 16.42 & 215.72 & sleek -orange \\
\hline L1t1-9 & 125 & 124 & 126 & 115.25 & 20.22 & 112.07 & sleek - red \\
\hline L1t2-4 & 127 & 126 & 127 & 39.73 & 55.69 & 123.99 & sleek- yellow \\
\hline L1t2-6 & 125 & 127 & 126 & 23.32 & 84.24 & 137.39 & sleek- yellow \\
\hline L1t2-7 & 126 & 126 & 125 & 103.70 & 52.88 & 78.15 & sleek - yellow \\
\hline L1t2-11 & 127 & 126 & 127 & 120.09 & 64.56 & 86.40 & sleek - yellow \\
\hline L1t3-3 & 126 & 125 & 126 & 104.64 & 21.12 & 133.99 & thorns - orange \\
\hline L2t1-5 & 127 & 125 & 125 & 157.65 & 30.47 & 145.86 & sleek - yellow \\
\hline L2t1-11 & 128 & 126 & 126 & 102.35 & 24.46 & 100.80 & sleek -yellow \\
\hline L2t1-16 & 127 & 125 & 125 & 153.22 & 27.65 & 90.24 & sleek - yellow \\
\hline L2t1-20 & 126 & 126 & 126 & 214.21 & 32.63 & 87.71 & sleek - yellow \\
\hline L2t2-5 & 125 & 126 & 127 & 107.00 & 66.04 & 145.73 & thorns yellow \\
\hline L2t2-13 & 125 & 126 & 128 & 132.58 & 31.21 & 58.42 & sleek- yellow \\
\hline L2t2-19 & 126 & 126 & 127 & 247.60 & 34.23 & 118.57 & thorns- yellow \\
\hline L2t2-20 & 126 & 127 & 127 & 307.30 & 32.18 & 130.64 & thorns- yellow \\
\hline L2t3-2 & 126 & 127 & 127 & 93.42 & 14.47 & 202.13 & sleek- orange \\
\hline L2t3-6 & 126 & 127 & 128 & 107.21 & 29.56 & 183.5 & thorns- yellow \\
\hline L2t3-9 & 127 & 128 & 127 & 77.30 & 22.84 & 251.36 & thorns - yellow \\
\hline L2t1-1 & 129 & 128 & 128 & 85.80 & 74.33 & 145.33 & thorns orange \\
\hline L2t1-5 & 129 & 129 & 129 & 57.13 & 53.59 & 140.33 & thorns - yellow \\
\hline L2t2-4 & 128 & 129 & 130 & 97.54 & 16.09 & 154.33 & sleek -yellow \\
\hline L2t3-1 & 126 & 126 & 127 & 139.98 & 33.73 & 50.50 & sleek -orange \\
\hline L2t3-3 & 127 & 126 & 128 & 102.00 & 26.59 & 78.53 & thorns -yellow \\
\hline regression coefficients & 0.599 & 0.70 & & -0.10 & -0.49 & \\
\hline & & & & & & & \\
\hline
\end{tabular}

\section{Conclusion:}

Using of different mutagen treatment was effective tools to obtained new safflower genotypes, spineless, earliness and high seed yield. We can used this new genotypes in breeding program to obtain new varieties are suitable for cultivation at reclaimed desert lands as a new oil crops in Egyptian agriculture.

\section{Reference}

Ahmad, M.S. (2011). A new technique for induction of mutations in plant (induction of mutation in bread wheat) Egypt. J. Plant Breed(15) 2: 193-205.
Dhole, V. J.; J.J. Maheshwari and Shanti Patil (2003). Studies on mutations induced by EMS in soybean (Glycinemax L.) Agricultural Science Digest, 23(3): 226-228.

Fahmy, E.M.; M.A. Rashed; M.T.M. Sharabash and A.H.A. Hammad (1997). "Effect of gamma rays on yield and its components for some soybean cultivars (Glycine max L. Merill)". Arab Universities Journal of Agricultural Sciences, 5: 57-68.

FAOSTAT, (2006). FAO Statistical Databases, http://apps.fao.org.

Geetha, K. and P. Vaidyanathan (1998). "Studies on induction of mutations in soybean (Glycine max L. Merill) 
through physical and chemical mutagens". Agricultural- Sciences Digest Karnal, 18:27-30.

Hajduch, M.; F. Debre.; B. Bohmova and B. Pretova (1999). "Effect of different mutagenic treatments on morphological traits of M2 generation of soybean". Soybean Genetics Newsletter .March 4pp; accessible via the World Wide Web at http:www.Soygenetics.Org. International Letters of Natural Sciences; 42:76-82.

Hassan, S.; M.A. Javed.; S.U.K. Khattak and M.M. Iqal (2001). "A high yielding better quality chickpea mutant variety (NIFA-95)". Mutation Breeding Newsletter, 45:6-7.

Kharkwal, M.C. (2000). "Induced mutations in Chickpea (Cicer arietinum L.) IV. Types of macromutation induced". Indian Journal of Genetics, 60:305-320.

Mia, M.F.U. and M.A.Q. Shaikh (1997). Induction of large seeded mutants in groundnut (Arachis hypogaea L.) SABRAOJ, 29:103-104.

Mihov, M.; A. Mehandjiev and M. Stoyanova (2001)."Mutagenesis as a breeding method in lentil". Mutation Breeding Newsletter, 45:3234.

Sheeba, A.; J. Anbumalarmathi. ; S. Babu and S.M. Ibrahim. (2005).
Mutagenic effect of gamma rays and EMS in M1generation in sesame (Sesamum indicum L.). Research on Crops, 6:303-306.

Solanki, I.S. and B. Sharma (1999). "Induction and exploitation of polygenic variability in lentil". Journal of Genetics and Breeding, 53: 79 86.

Soliman, S.S.A.; M.S. Eisa.; T.A. Ismail.; A. Nadia.; Naguib and F.E Azza (2003). "Induction of salt tolerance mutants in Faba bean (Vicia $f a b a$ L.). Promising line mutants under saline and normal soil condition". Zagazig J. Agric. Res, 30:213-229.

Sujatha, M. (2007). "Advances in safflower biotechnology". Functional Plant Science and Biotechnology. Global Science Books. Ed. Texeira Da Silva, 1:160-170.

Velasco, L.; B. Perez-Vich and J.M. Fernandez-Martinez (2005). "Identification and genetic characterization of a safflower mutant with a modified tocopherol profile". Plant Breeding, 124:459-463.

Wani, A.A. and M. Anis (2001). "Gamma rays induced bold seeded high yielding mutant in Chickpea". Mutation Breeding Newsletter, 45:20-21. 
إستحداث الطفرات فى بعض الطرز الوراثية للقرطم

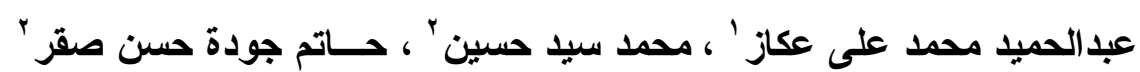

'قسم المحاصيل - كلية الزراعة - جامعة الأزهر - القاهرة.

"قام المحاصيل - كلية الزراعة - جامعة الأزهر بأسيوط.

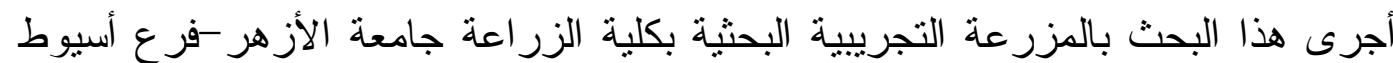

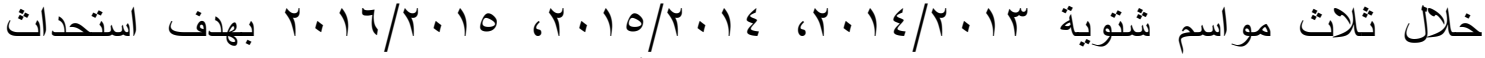

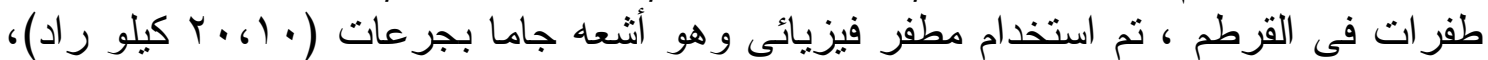

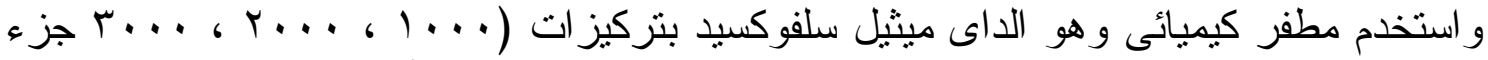

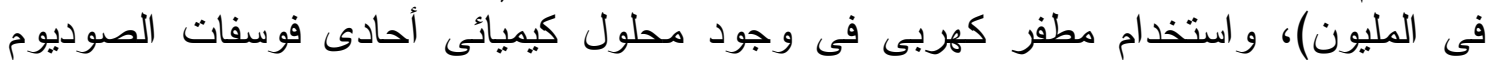

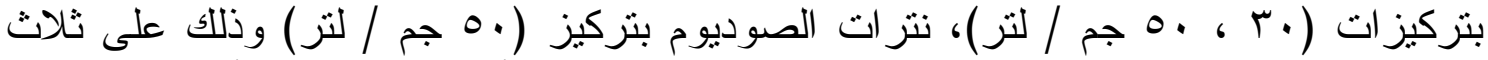

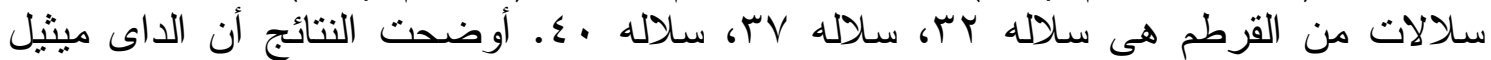

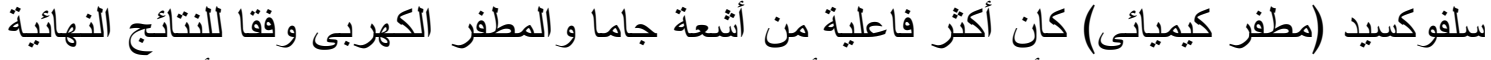

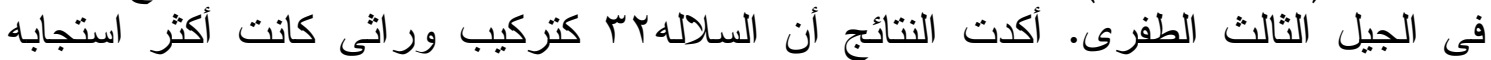

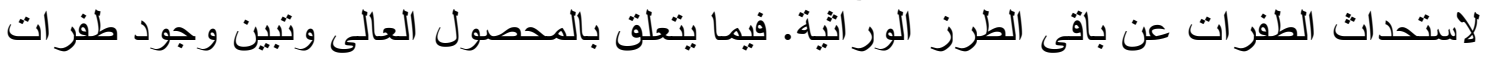
عديمة الأشو الك، مبكرة عن الطر الثر الأبوري.

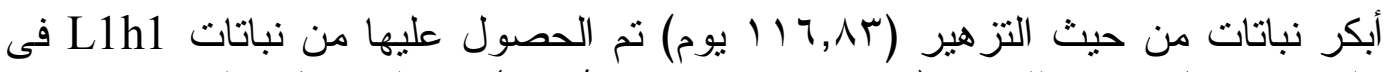

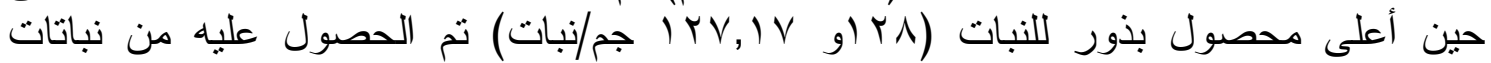

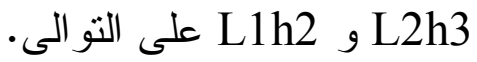

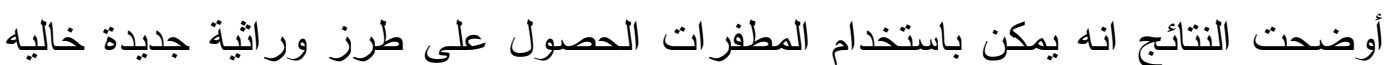

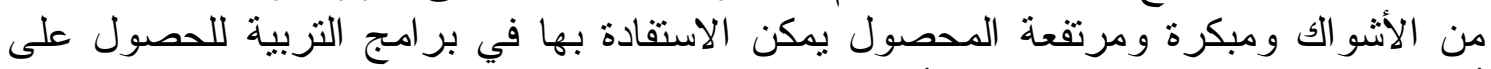
أصناف جديدة تصلح للزر اعة فى الأر اضي الصحر اوية المستصلحة. 